\title{
Identification and Verification of the Effect of miR- 143 on the Cardioprotective Role of Phosphocreatine in Doxorubicin- Induced Cardiotoxicity by Integrated Bioinformatics Analysis
}

\section{Chi Zhou}

School of Pharmacy, Xianning Medical College, Hubei University of Science and Technology

\section{Zi-Mo Zhou}

Hubei Key Laboratory of Diabetes and Angiopathy, Medicine Research Institute, Xianning Medical College, Hubei University of Science and Technology

\section{Ling $\mathrm{Hu}$}

Hubei Key Laboratory of Diabetes and Angiopathy, Medicine Research Institute, Xianning Medical College, Hubei University of Science and Technology

\section{Ya-Yuan Yang}

Hubei Key Laboratory of Diabetec and Angiopathy, Medicine Research Institute, Xianning Medical College, Hubei University of Science and Technology

\section{Xiang-Wen Meng}

Hubei Key Laboratory of Diabetes and Angiopathy, Medicine Research Institute, Xianning Medical College, Hubei University of Science and Technology

\section{Xiao-Song Yang ( $\nabla$ xiaosong_yang@hbust.edu.cn )}

Xianning Medical College: Hubei University of Science and Technology Faculty of Medicine https://orcid.org/0000-0002-3929-1736

\section{Research Article}

Keywords: doxorubicin, cardiotoxicity, oxidative stress, miRNA, bioinformatic analysis.

Posted Date: December 30th, 2021

DOI: https://doi.org/10.21203/rs.3.rs-1168472/v1

License: (c) (1) This work is licensed under a Creative Commons Attribution 4.0 International License. Read Full License 


\section{Abstract}

Purpose MicroRNAs (miRNAs) have been reported to play pivotal role in drugs-induced cardiotoxicity act as biomarkes, diagnostic tools and endogenous repressors of gene expression by lowering mRNA stability and interfering with mRNA translation. However, the effect of miRNAs on doxorubicin-induced cardiotoxicity still not clear. In the present study, we identified several key candidate miRNAs involving doxorubicin (DOX)-induced cardiotoxicity in rat myocardial tissues and adult rat cardiomyocytes from the Gene Expression Omnibus (GEO) database via integrated bioinformatics analysis, and the possible effect of miR-143 in the protection of DOX-induced cardiotoxicity by phosphocreatine was subsequently investigated in vivo and in vitro.

Methods GSE36239 miRNA expression profiles of DOX-induced cardiotoxicity in rat myocardial tissues and adult rat cardiomyocytes (ARC) were extracted fromGEO datasets. $\left|\log _{2} \mathrm{FC}\right|>1$ and $P<0.05$ were set as screening criteria, miRNAs expressed in myocardial tissues or ARC were selected as different expression miRNA (DEMs), and subsequently the key miRNAs were obtained from candidate DEMs between myocardial tissues and ARC with Venny 2.1 software. Target genes of miR-143 were predicted with Targetscan and miRBase in the species of homo sapiens, and candidate genes were obtained with Venny 2.1. The gene ontology (GO) and Kyoto Encyclopedia of Genes and Genomes pathway (KEGG) enrichment analyses were carried out. Final, the expression and potential role of miR-143 were verified in DOX-induced cardiotoxicity of rat and cardiomyocytes H9c2.

Results A total 24 DEMs were captured, including 15 up-regulated and 9 down-regulated genes in rat myocardial tissues and 42 DEMs were discovered, including 13 up-regulated and 29 down-regulated in ARC. Ultimately, 6 DEMs were determined in rat myocardial tissues and ARC by venny 2.1 software. 46 target genes of miR-143, one of the 6 DEMs, were captured from the predict results of Targetscan and miRBase with venny 2.1. The target genes were notably enriched in biological processes (BP) such as cell proliferation and migration. KEGG pathway analysis showed the target genes were enriched in HIF-1 and PI3K-Akt signaling pathway, which closely related to the oxidative stress and cardiomyocytes apoptosis. Further, western blot and RT-PCR results showed DOX-induced oxidative stress down-regulated the expression of miR-143 and Nrf2, SOD and BCL2, and up-regulated Bax and Cleaved caspase 3, while they could been reversed by the intervention of phosphocreatine (PCr) or N-acetyl-L-cystine (NAC) in DOXinduced cardiotoxicity in vivo and in vitro.

Conclusion Our data showed that DOX-induced oxidative stress could decrease the expression of miR143 , promote apoptosis of cardiomyocytes, while PCr or NAC mediated antioxidation could reverse the expression down-regulation of miR-143, alleviated apoptosis in DOX-induced cardiotoxicity. Our findings elucidated the regulatory network involving miR-143 in DOX-induced cardiotoxicity, and might unveiled a potential biomarker and molecular mechanisms, which could be helpful to the diagnosis and treatment of DOX-induced cardiotoxicity.

\section{Introduction}


In recent years, survival rates for cancer patients are steadily increasing due to advanced targeted therapies and early intervention against the side effects of chemotherapy [1]. Doxorubicin (DOX), as an anthracycline derived from Streptomyces peucetius, is one of the most effective and widely used in the clinical treatment of a variety of solid or circulatory malignancies [2]. However, it is also limited in clinical application due to its serious toxic and side effects, mainly manifested as irreversible degenerative dilated cardiomyopathy, arrhythmia, eventually caused congestive heart failure, which seriously threaten the survival of tumor patients and affecting the results of long-term anti-tumor therapy $[3,4]$. Investigating the underlying molecular mechanisms, strategies and exploring biomarkers for early diagnosis to limit DOX-induced cardiotoxicity might have important clinical implications. Previous studies have extensively discussed the mechanisms of DOX-induced cardiotoxicity, involving DOX induced DNA damage, excessive production of reactive oxygen species (ROS), mitochondrial and energy metabolism dysfunction, endoplasmic reticulum stress, disturbance of calcium homeostasis, death mediated by programmed necrosis, coke death, iron necrosis [4-7]. However, the precise mechanism is still elusive.

MicroRNAs (miRNAs)are a class of small non-coding RNAs composed of 21-25 nucleotides, which regulates gene expression at the post-transcriptional level by inhibiting mRNA translation or promoting its degradation [8], play an important role in many pathophysiological processes such as cell proliferation, apoptosis, necrosis, differentiation and metabolism $[9,10]$. Emerging studies have shown the importance of miRNA in various human diseases including DOX-induced cardiotoxicity. Recent, miRNA expression profiling of hear tissues in mouse or rat models of DOX-induced cardiotoxicity and plasma of cancer patient after chemotherapy with DOX have been investigated by miRNA array, and numerous difference expression miRNA were reported at the early stage of DOX treatment [11-13]. These data implied that miRNA possesses potential clinical applicability as biomarkers and intervention targets.

Our previous studies reported that DOX-induced oxidative stress promoted cardiomyocytes apoptosis and necroptosis via transforming growth factor beta-activated kinase 1 (TAK1) signaling pathway, while the intervention of $\mathrm{PCr}$ with antioxidant activity attenuated oxidative stress, ameliorated DOX-induced apoptosis and necroptosis by activating the TAK1 signaling pathway [14]. In the present study, GSE36239 extracted from GEO database was used to analyze miRNA expression changes by online tool GEO2R in DOX -induced cardiotoxicity in vivo and in vitro, and 6 different expression miRNAs (DEMs) were captured. Furthermore, target genes of miR-143 were used to perform the Gene Ontology (GO) and Kyoto Encyclopedia of Genes and Genomes pathway (KEGG) enrichment analyses. The results showed that the target genes of miR-143 were associated with cell proliferation, survival and HIF-1a and PI3K-Akt signaling pathway, which were closely related to oxidative stress and apoptosis in cardiomyocytes [15, 16]. Our experimental data confirmed that DOX down-regulated the expression of miR-143, while PCr or antioxidant NAC alleviated the expression down-regulation of miR-143 and attenuated apoptosis induced by DOX.

\section{Material And Methods}


Based on miRNA expression profile data of GSE36239 obtained from GEO database, 4 groups of raw data files from 12 samples were downloaded, including Rat normal control group (Rat CON, $n=3$ ) and Rat DOX treatment group (Rat DOX, $n=3$ ), ARC control group (ARC CON, $n=3$ ) and ARC DOX treatment group (ARC DOX, $n=3$ ), GSE36239 was based on GPL15295 platform Rat miRNA nCounter Expression Assay Kit.

\section{Dems Identification}

According to GEO2R (http://www.ncbi.nlm.nih.gov/geo/geo2r) online software analysis, $P<0.5$ and $\| \log _{2} \mathrm{FC} \mid>1$ were set as filter condition, DEMs and their corresponding the fragments per kilobase of exon per million (FPKM) were captured on DOX-induced cardiotoxicity in rat heart and adult rat cardiomyocytes (ARC), respectively. The DEMs were shown as heatmaps with the Clustvis online tool. The final DEMs were obtained from the intersection of DEMs between the group rat hearts and ARC through online software Venny 2.1. (http://bioinfogp.cnb.csic.es/tools/venny/index/html).

\section{Prediction and functional enrichment analysis on the target genes of miR-143}

Targetscan (http://www.targetscan.org/vert_72/) and miRBase (http://www.mirbase.org/) online software were used to predict target genes of miRNA in species Homo sapiens, Venny 2.1 software was used to map the intersection of target genes predicted by different online prediction software. GO and KEGG pathway enrichment analysis on target mRNAs of miRNA were performed by DAVID data platform (http://david.ncifcrf.gov/tools.jsp). GO analysis included terms in the biological process, molecular function function and cellular component categories. $P<0.05$ was considered to indicate significant enrichment.

\section{Experimental Animals}

The establishment on rat model of DOX-induced cardiotoxicity have been described in our previous literature, in which, cardiac function and histopathological parameters were shown [14]. In the present study, the total RNA from rat heart tissues were used to analyse the expression of miR-143 by RT-PCR.

\section{$\mathrm{H} 9 \mathrm{c} 2$ cells culture and experimental treatment}

H9c2 cells were purchased from FuDan IBS Cell Resource Center of Fudan University, (Shanghai, China) and cultured in Dulbecco's modified Eagle's medium (11995-055, Gibco) contained 10\% fetal bovine serum (16000-044) and 1\% penicillin (100 units $\left.\cdot \mathrm{mL}^{-1}\right) /$ streptomycin $\left(100\right.$ units $\left.\cdot \mathrm{mL}^{-1}\right)$ in the incubator with $5 \% \mathrm{CO}_{2}$ and $37^{\circ} \mathrm{C}$. Upon reaching $70 \% \varangle 80 \%$ confluence, the cells were pretreated with $\mathrm{PCr}(0.5 \mathrm{mM}$, P7936-5G, Sigma-Aldrich) or NAC (0.5 mM, S0077, Beyotime) for $1 \mathrm{~h}$, and then exposed to DOX (1 $\mu \mathrm{M})$ for $24 \mathrm{~h}$.

\section{Total Rna Extraction And Real-time Pcr}

Total RNA was extracted from $\mathrm{H} 9 \mathrm{c} 2$ cells or rat hearts with TRIzol reagent, the concentration and purity were determined with nanodrop 2000 (Thermo Scientific, USA). The total mRNA was reversely transcribed 
into cDNA with stem-loop primer by Hiscript II 1st strand cDNA Synthesis kit (Vazyme Biotech, Nanjing, China). Quantitative RT-PCR reaction was carried out using CFX Connect Real-Time Systerm (BIO-RAD, California, MA, USA) with AceQ qPCR SYBR Green Master Mix (Vazyme Biotech, Nanjing, China). The procedure of amplification reaction was as follow: $95^{\circ} \mathrm{C}$ for $5 \mathrm{~min}$, then 40 cycles of $95^{\circ} \mathrm{C}$ for $10 \mathrm{~s}$ and $60^{\circ} \mathrm{C}$ for $30 \mathrm{~s}$. U6 was selected as endogenuous reference gene and the relative expression levels was analyzed using $2^{-\triangle \triangle} \mathrm{CT}$ method. All the sequence of primers were presented in Table.1.

\section{Tab.1 Sequence Of Primers For Rt-pcr}

\begin{tabular}{|c|c|c|}
\hline Gene & Primer sequence $\left(5^{\prime}-3^{\prime}\right)$ & Length/bp \\
\hline UDP & R: 5'-CAGTGCGTGTCGTGGAGT-3' & 18 \\
\hline $\begin{array}{l}\text { rno- } \\
\text { miR- } \\
143- \\
\text { SLP }\end{array}$ & $\begin{array}{l}\text { 5'- } \\
\text { GTCGTATCCAGTGCGTGTCGTGGAGTCGGCAATTGCACTGGATACGACTGAGCT- } \\
3^{\prime}\end{array}$ & 54 \\
\hline $\begin{array}{l}\text { rno- } \\
\text { miR- } \\
143- \\
5 p\end{array}$ & F: 5'-GGTGCAGTGCTGCATCTCTG-3' & 20 \\
\hline U6 & F: 5'-GCTTCGGCAGCACATATACTAA-3' & 22 \\
\hline U6 & R: 5'-AACGCTTCACGAATTTGCGT-3' & 20 \\
\hline
\end{tabular}

\section{Statistical analysis}

All experimental data were expressed as mean \pm SEM. The software GraphPad Prism 5 Project (GraphPad Software, San Diego, CA) was used for data statistics. One-way ANOVA and T-test were used for data analysis.And Statistical significance was detected at the $P<0.05$.

\section{Result}

\section{Analysis and acquisition of DEMs}

The data of GSE36239 miRNA expression profiles, which was based on GPL15295 Rat miRNA nCounter Expression Assay Kit, were extracted from the GEO database. The GSE36239 cohort comprised 15 samples, including 5 groups. The overall flow diagram of our research is presented in Figure 1. In this study, only the miRNA expression profiles of 4 groups, including the heart of 3 DOX-treated rats and that of 3 controls, 3 DOX-treated ARC and that of 3 control, were used to difference analysis. Using $P<0.05$ and $|\log 2 \mathrm{FC}|>1$ as threshold cutoff point, 24 DEMs were highlighted in the gourps of DOX-treated heart 
and control. Of these, 15 DEMs were up-regulated and 9 DEMs were down-regulated. On the other hand, 42 DEMs were highlighted in the groups of DOX-treated ARC and control. Of these, 13 DEMs were upregulated and 29 DEMs were down-regulated. Two heat maps of the 24 and 42 DEMs from the goups of rat hearts and ARC were shown in Figure 2A and 2B, respectively. Online software Venny 2.1 was used to map the intersection of DEMs between the groups of rat hearts and ARC. Final, a total of 6 overlapping DEMs were obtained ( Figure.2C), while the miR-3562 was excluded due to the opposite expression trends in rat hearts and ARC. Therefore, the DEMs involving miR-29b, miR-143, miR-133b, miR-29c, miR-30a and miR-30e were finally identified.

\section{The expression profiles of DEMs}

The expression changes of the candidate 6 DEMs from GSE36239 were shown in Figure 3A-G, all the 6 DEMs were down-regulated in the heart of DOX-treated rats and DOX-treated ARC compared with each corresponding control group. On the other hand, the effect of miR-29b, miR-133b, miR-29c, miR-30a and miR-30e in DOX-induced cardiotoxicity have been reported [17-20], the expression trends were consistent with the data from GSE36239. While it was reported that miR-143 were up-regulated and promoted cardiotoxicity in a mouse model of DOX-induced cardiotoxicity[21], this result is contrary to the data from GSE36239. To further determination of the role of miR-143 in DOX-induced cardiotoxicity, the expression of miR-143 was analyzed in the heart of rats in DOX-induced cardiotoxicity by RT-PCR. In the model, the data on echocardiography and pathological changes of heart tissue have been reported in our previous literature [14]. However, the result on RT-PCR detection showed that DOX significantly down-regulated the expression of miR-143, which was consistent with the data from GSE36239. Further, our data also showed that the intervention of PCr drastically reversed the down-regulation of miR-143 induced by DOX (Figure $3 \mathrm{H}$ ). The above results indicated that miR-143 maybe play am important regulatory role in DOXinduced cardiotoxicity.

\section{Prediction On Target Genes Of Mir-143}

According to the above analysis, potential target genes of miR-143 were predicted by online software Targetscan7.2 and miRBase. The 46 target genes were captured and shown in the intervention of only one target gene of miR-143 obtained was MAPK7 with miRBase in rat. Therefore, the predicted results of rat miR-143 in miRBase will not be considered in furture analysis. As shown in Figure .4, 160 candidate target genes were predicted by miRBase online software and 497 candidate target genes were predicted with TargetScan online software. Target genes were mapped by online software Venny 2.1, 46 target genes were shown in the intersection (Figure 4A).

\section{Enrichment Analysis Of Go And Kegg Pathway}


To understand the potential functions mechanisms of miR-143 in DOX-induced cardiotoxicity, GO and KEGG pathway enrichment analysis were performed based on the 46 predicted target genes with DAVID online database. The value of $\mathrm{P}$ severed as the evaluation scale, the result of enrichment analysis showed that predicted target genes in BP were mainly enriched in the positive regulation of cell proliferation, positive regulation of cell migration, response to estradiol and cellular response to hypoxia. In CC, predicted target genes were principally enriched in the cytosol, Golgi apparatus. The last, in MF, predicted target genes were enriched in the ATP binding, transmembrane receptor protein tyrosine kinase activity, identical protein binding and protein kinase activity. KEGG pathway analysis revealed that the target genes were mainly associated with HIF-1 signaling pathway, PI3K-Akt signaling pathway and FoxO signaling pathway. According to the existing literature, the signaling pathways all revealed closely related to oxidative stress and apoptosis of cardiomyocytes and played an important role occurrence and development of cardiomyopathy [22-24]. In this study, the KEGG enrichment for HIF-1 signaling pathway was revealed (Figure 4B-E).

\section{Effect of PCr on oxidative stress and apoptosis induced by DOX in $\mathrm{H} 9 \mathrm{c} 2$ cells}

Our previous studies have reported that DOX promoted oxidative stress by down-regulating antioxidant activity of cardiomyocytes, which is an important factor in myocardial injury induced by DOX [14, 25]. PCr, acting as an clinical drug, significantly promoted the expression of nuclear factor-erythroid 2-related factor 2(Nrf2) and superoxide dismutase (SOD), improved oxidative stress and alleviated cardiomyocytes apoptosis induced by DOX in the heart of rat and H9c2 cells. Based on previous research, in this study, the fact was further validated that DOX triggered down-regulation expression of Nrf2 and SOD and Bcl2, while promoted up-regulation of Bax and C-Caspase 3. The intervention of PCr significantly alleviated the expression trend induced by DOX (Figure $5 \mathrm{~A}$ and $\mathrm{B}$ ). The result demonstrated again that DOX induced oxidative stress resulting in apoptosis of $\mathrm{H} 9 \mathrm{c} 2$ cells. PCr ameliorated oxidative stress to attenuated apoptosis induced by DOX in H9c2 cells.

\section{Effect of PCr and NAC on the expression of miR-143 induced by DOX in $\mathrm{H} 9 \mathrm{c} 2$ cells}

The effect of PCr on the expression of miR-143 induced by DOX in the heart of rat had been analyzed, we further verified the effect of DOX on the expression of miR-143 in H9c2 cells and evaluated intervention effect of PCr. The result showed that DOX significantly down-regulated expression of miR-143, while the intervention of PCr obviously inhibited the down-regulation expression of miR-143 induced by DOX in H9c2 cells (Figure 6A). On the other hand, our previous study had reported that antioxidants NAC significantly improved oxidative stress and inhibited apoptosis and necroptosis induced by DOX in H9c2 cells[14]. In present study, NAC treatment up-regulated the expression of miR-143 and reversed the downregulation expression of miR-143 induced by DOX in H9c2 cells (Figure 6B). The above data indicated 
during DOX cardiotoxicity that DOX mediated oxidative stress promoted apoptosis and necroptosis, which was negatively correlated with the expression of miR-143. In the meantime, PCr mediated antioxidant effect attenuated apoptosis and necroptosis induced by DOX, which was positively correlated with the expression of miR-143.

\section{Discussion}

In the past 20 years, cancer-related mortality have continuously declined, however, cancer is still a major leading cause of death worldwide [26]. DOX is most effective chemotherapeutic drug for a wide range of cancers, but the dose-dependent, unpredictable cardiotoxicity effect of DOX is still a highly concerning issue. Although the intervention methodology and strategy have greatly improved over the years, DOXinduced cardiotoxicity remain a serious risk to survival in cancer patients. So, it is very important to clarify the mechanism and explore more sensitive and predictive detection methods on early pre-symptomatic diagnostic and treatment of DOX-induced cardiotoxicity. Our previous study found that TAK mediated apoptosis and necroptosis of cardiomyocytes, which were novel mechanism and maybe important drug intervention targets on DOX-induced cardiomyoxicity.

In current study, GSE36239 from GEO database showed the expression profile of miRNA on DOX-induced cardiotoxicity in vivo and in vitro. Analysis data with GEO2R online software revealed that 6 candidates miRNA, which showed significant difference expression and consistent change trends between DOX treatment group and conrol group in vivo and in vitro, were determined involving miR-29b, miR-133b, miR29c, miR-30a, miR-30e and miR-143. Among the DEMs, some have been reported which performed an important regulatory role in DOX-induced cardiotoxicity. Jing XB et al reported that miR-29 mitigated DOXinduced myocardial apoptosis by targeting Bax [17], Roca-Alonso L et al reported that miR-30 targeted pro-apoptotic gene BNIP3L/NIX protected cardiotoxicity induced by DOX [19], Li Z et al reported that miR133b alleviated DOX-induced cardiomyocyte apoptosis and cardiac fibrosis by targeting PTBP1 and TAGLN2 [18]. However, miR-143 expression profile from GSE36239 was opposite with the data reported by Li XQ et al [27]. Therefore, miR-143 was further investigated in the heart tissues from our previous research on DOX-induced cardiotoxicity in rats. Our experimental data showed that the expression of miR143 was significantly down-regulated induced by DOX in the heart of rats, which was consistent with expression profile from GSE36239. While the intervention of PCr with antioxidant activity drastically reversed down-regulation expression of miR-143 induced by DOX in the heart of rats.

GO and KEGG enrichment analysis of miR-143 target genes implied that the signaling pathways revealed closely related to oxidative stress and apoptosis of cardiomyocytes. On DOX-induced cardiotoxicity in rat, our experimental result showed that PCr with antioxidation significantly up-regulated miR-143 expression. Further verification was performed in $\mathrm{H} 9 \mathrm{c} 2$ cells, the result indicated that the pre-treatment of $\mathrm{PCr}$ or antioxidant NAC significantly attenuated the down-regulation expression of antioxidant cytokines Nrf2 and SOD, and ameliorated apoptosis induced by DOX. The expression of miR-143 was also investigated that DOX decreased the expression of miR-143, PCr or NAC drastically reversed the expression of miR-143 induced by DOX, respectively. The results further implied that miR-143 possessed an important role in 
DOX-induced cardiotoxicity, the regulatory mechanism maybe closely related with oxidative stress induced by DOX.

In conclusion, 6 DEMs from GSE36239 were selected with filter condition of $\mathrm{P}<0.05$ and $\left|\log _{2} \mathrm{FC}\right|>1$ on DOX-induced cardiotoxicity in rat, and the expression profiles were shown. In this study, the expression profile of miR-143, one of the DEMs, was also verified in vivo and in vitro. More importantly, the potential functions of miR-143 were identified by bioinformatics, which was closely related to oxidative stress and apoptosis induced by DOX in cardiomyocytes. These findings also provide novel insight into the potentially pathogenesis and association with presymptomatic DOX-induced cardiotoxicity during the chemotherapy. On the other hand, this findings may be valuable in identifying the risk of patients for the development of DOX-induced cardiotoxicity and thus to avoid the accumulating toxic effects from the subsequent doses during chemotherapy. A larger cohort of patient is needed to confirm the value of these potential biomarkers for presymptomatic DOX-induced cardiotoxicity and the regulatory mechanism used as target for the clinical intervention in future studies.

\section{Abbreviations}

DOX doxorubicin

ARC adult rat cardiomyocytes

miRNAs microRNAs

GEO Gene Expression Omnibus

DEMs different expression miRNAs

KEGG Kyoto Encyclopedia of Genes and Genomes pathway

PCr phosphocreatine

NAC N-acetyl-L-cystine

\section{Declarations}

Author Contributions Xiao-Song Yang and Xiang-Wen Meng conceived and designed the study. Zi-Mo Zhou, Chi Zhou and Ya-Yuan Yang conducted the experiments. Xiao-Song Yang and Ling Hu analyzed the data. Xiao-Song Yang and Xiang-Wen Meng wrote the manuscript. All authors read and approved the manuscript.

Funding This work was supported by Hubei Institutions of Higher Learning Outstanding Young Science and Technology Innovation Team Project, Hubei Provincial Department of Education Grant (T201921), Natural Science Foundation, Science and Technology Bureau of Hubei Province (2021CFB439), Scientific 
Research Program of Hubei Provincial Department of Education Grant (B2021228), National Science Foundation Project Cultivation Program of Hubei University of Science and Technology Grant (202022GP05), Special Topics Project of Hubei Key Laboratory of Diabetes and Angiopathy Grant (2020XZ09) and Special Topics Project of School of Stomatology and Ophthalmology, Xianning Medical College, Hubei University of Science and Technology (2021WG07).

Data Availability Author can confirm that all relevant data are included in the article and/or its supplementary information files.

Ethical Approval and Consent to participate All applicable international, national, and/or institutional guidelines for the care and use of animals were followed. This article does not contain any studies with human participants.

Consent for Publication All authors approved its publication.

Conflict of Interest The authors declare no conflicts of interest.

\section{References}

1. Miller KD, Siegel RL, Lin CC, et al. Cancer treatment and survivoship statistics, 2016. CA Cancer J Clin. 2016;66(4):271-89

2. Gioffre S, Ricci V, Vavassori C, et al. Plasmatic and chamber-specific modulation of cardiac microRNAs in an acute model of DOX-induced cardiotoxicity. Biomed Pharmacother. 2019;110:1-8

3. Lefrak EA, Pitha J, Rosenheim S, Gottlieb JA. A clinicopathologic analysis of adriamycin cardiotoxicity. Cancer. 1973;32(2):302-14

4. Zhang S, Liu X, Bawa-Khalfe T, et al. Identification of the molecular basis of doxorubicin-induced cardiotoxicity. Nat Med. 2012;18:1639-42

5. Carvalho FS, Burgeiro A, Garcia R, Moreno AJ, Carvalho RA, Oliveira PJ. Doxorubicin-induced cardiotoxicity: from bioenergetic failure and cell death to cardiomyopathy. Med Res Rev. 2014;34(1):106-35

6. Tadokoro $\mathrm{T}$, Ikeda $\mathrm{M}$, Ide $\mathrm{T}$, et al. Mitochondria-dependent ferroptosis plays a pivotal role in doxorubicin cardiotoxicity. JCI Insight. 2020;5(9):e132747

7. Christidi E, Brunham LR. Regulated cell death pathways in doxorubicin-induced cardiotoxicity. Cell Death Dis. 2021;12(4):339

8. Winkle M, El-Daly SM, Fabbri M, Calin GA. Noncoding RNA therapeutics-challenges and potential solutions. Nat Rev Drug Discov. 2021;20(8):629-51

9. Mori MA, Ludwig RG, Garcia-Martin R, Brandao BB, Kahn CR. Extracellular miRNAs: From Biomarkers to Mediators of Physiology and Disease. Cell Metab. 2019;30(4):656-73

10. Zhou SS, Jin JP, Wang JQ, et al. miRNAs in cardiovascular diseases: potential biomarkers, therapeutic targets and challenges. Acta Pharmacol Sin. 2018;39(7):1073-84 
11. Todorova VK, Makhoul I, Wei J, Klimberg VS. Circulating miRNA Profiles of Doxorubicin-induced Cardiotoxicity in Breast Cancer Patients. Ann Clin Lab Sci. 2017;47(2):115-9

12. Pereira JD, Tosatti JAG, Simoes R, Luizon MR, Gomes KB, Alves MT. microRNAs associated to anthracycline-induced cardiotoxicity in women with breast cancer: a systematic review and pathway analysis. Biomed Pharmacother. 2020;131:110709

13. Fa HG, Chang WG, Zhang XJ, Xiao DD, Wang JX. Noncoding RNAs in doxorubicin-induced cardiotoxicity and their potential as biomarkers and therapeutic targets. Acta Pharmacol Sin. 2021;42(4):499-507

14. Wang $\mathrm{C}$, Hu L, Guo S, et al. Phosphocreatine attenuates doxorubicin-induced cardiotoxicity by inhibiting oxidative stress and activating TAK1 to promote myocardial survival in vivo and in vitro. Toxicology. 2021;460:152881

15. Paul S, Gangwar A, Bhargava K, Ahmad Y. D4F prophylaxis enables redox and energy homeostasis while preventing inflammation during hypoxia exposure. Biomed Pharmacother. 2021;133:111083

16. Shi J, Yu TX, Song K, et al. Dexmedetomidine ameliorates endotoxin-induced acute lung injury in vivo and in vitro by preserving mitochondrial dynamic equilibrium through the HIF-1a/HO-1 signaling pathway. Redox Biol. 2021;41:101954

17. Jing XB, Yang JX, Jiang L, Chen JH, Wang HY. MicroRNA-29b regulates the mitochondria-Dependent Apoptotic Pathway by Targeting Bax in Doxorubicin Cardiotoxicity. Cell Physiol Biochem. 2018;48(2):692-704

18. Li Z, Ye ZK, Ma JZ, Gu Q, Teng JZ, Gong XX. MicroRNA-133b alleviates doxorubicin-induced cardiomyocyte apoptosis and cardiac fibrosis by targeting PTBP1 and TAGLN2. Int J Mol Med. 2021;48(1):125

19. Zhang XN, Lv SC, Zhang WQ, et al. Shenmai injection improves doxorubicin cardiotoxicity via miR30a/Beclin 1. Biomed Pharmacother. 2021;139:111582

20. Lai L, Chen JZ, Wang NF, Zhu GJ, Duan X, Ling F. MiRNA-30e mediated cardioprotection of ACE2 in rats with Doxorubicin-induced heart failure through inhibiting cardiomyocytes autophagy. Life Sci. 2017;169:69-75

21. Li XQ, Liu YK, Yi J, et al. MicroRNA-143 Increases Oxidative Stress and Myocardial Cell Apoptosis in a Mouse Model of Doxorubicin-induced Cardiac Toxicity. Med Sci Monit. 2020;26:e920394

22. Hsieh SR, Hsu CS, Lu CH, Chen WC, Chiu CH, Liou YM. Epigallocatechin-3-gallate-mediated cardioprotection by Akt/GSK-3 $\beta$ /caveolin signalling in $\mathrm{H} 9 \mathrm{c} 2$ rat cardiomyoblasts. J Biomed Sci. 2013;20(1):86

23. Miyata T, Takizawa S, van Ypersele de Strihou C. Hypoxia.1.Intracellular sensors for oxygen and oxidative stress: novel therapeutic targets. Am J Physiol Cell Physiol. 2011;300(2):C226-31

24. Maiese K, Hou J, Chong ZZ, Shang YC. A fork in the path: Developing therapeutic inroads with FoxO proteins. Oxid Med Cell Longev. 2009;2(3):119-29

25. Zhang T, Zhang Y, Cui MY, et al. CaMKII is a RIP3 substrate mediating ischemia- and oxidative stressinduced myocardial necroptosis. Nat Med. 2016;22(2):175-82 
26. Siegel RL, Miller KD, Fuchs HE, Jemal A. Cancer Statistics, 2021. 2021;71(1):7-33

27. 27. Li XQ, Liu YK, Yi J, et al. MicroRNA-143 increases Oxidative Stress and Myocardial Cell Apoptosis in a Mouse Model of Doxorubicin-Induced Cardiac Toxicity. Med Sci Monit. 2020;26:e920394

\section{Figures}

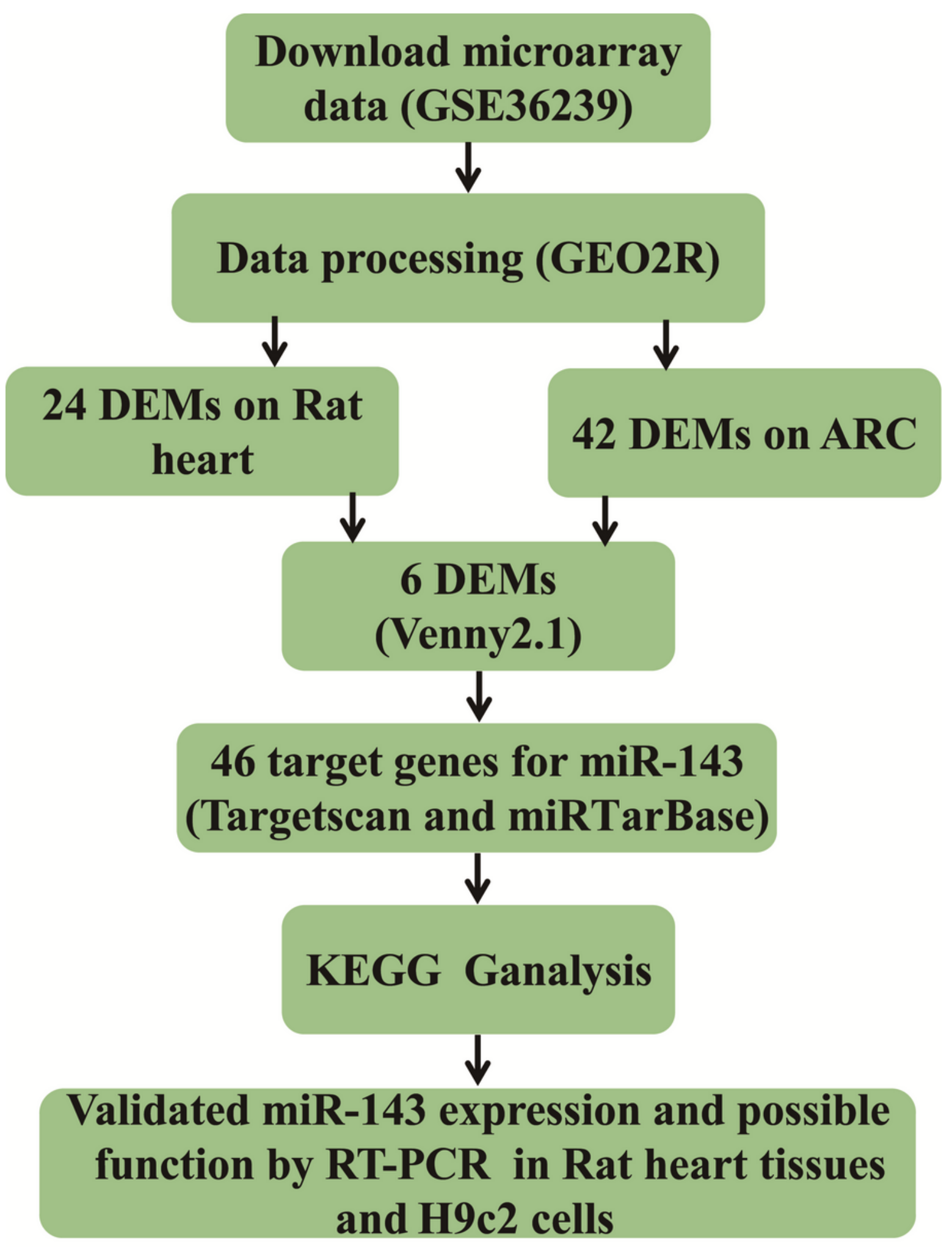


Figure 1

Flow diagram of the analysis procedure. Data collection, pre-processing, analysis and validation.

A

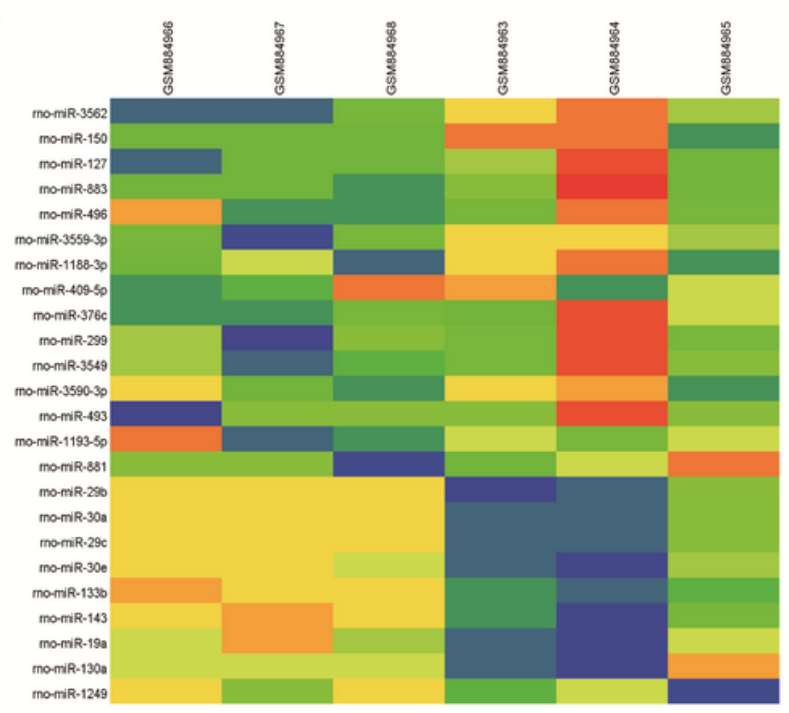

B

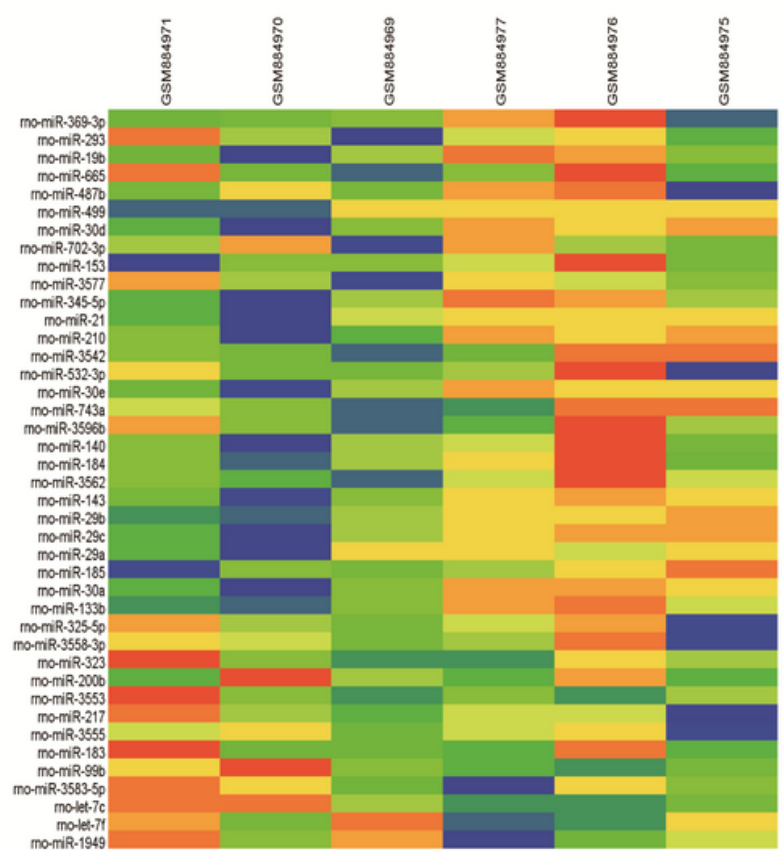

C

Rat heart

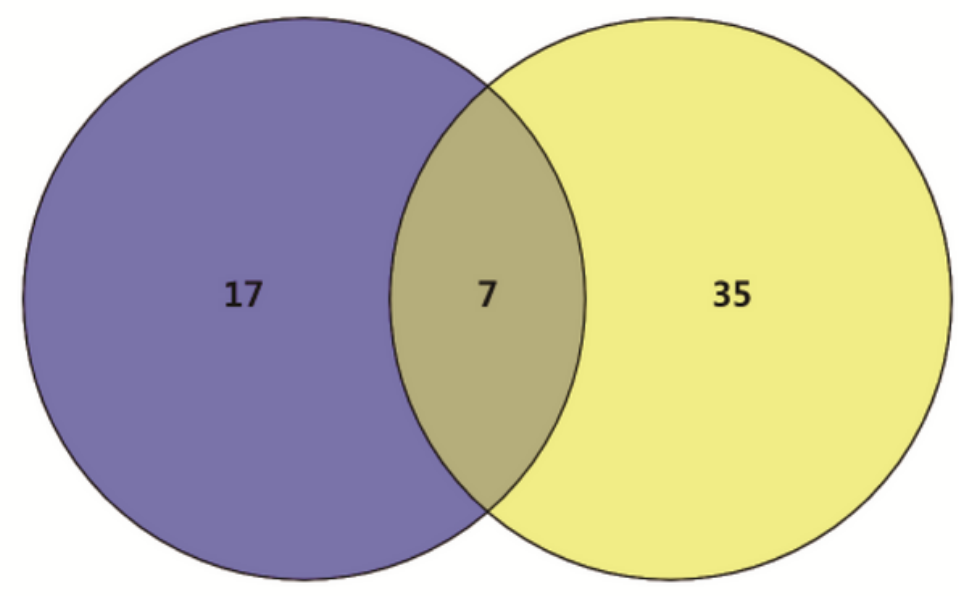

ARC

$\operatorname{miR}-29 b$

miR-29c

miR-30a

miR-30e

miR-143

miR-133b

$\operatorname{miR}-3562$

Figure 2

Identification of DEMs on DOX-induced cardiotoxicity in rat heart tissues and ARC. A Heat map of 15 upregulated DEMs and 9 downregulated in rat heart tissues; Samples of rat heart tissues: normal control group: GSM884966, GSM884967, GSM884968; DOX group: GSM884963, GSM884964, GSM884965. B Heat map of 13 up-regulated DEMs and 29 down-regulated DEMs in ARC. Samples of ARC, normal control group: GSM884975, GSM884976, GSM884977; DOX group: GSM884969, GSM884970, GSM884971. C Map the intersection of expression difference analysis of rat heart tissues and ARC by Venny 2.1. 
A

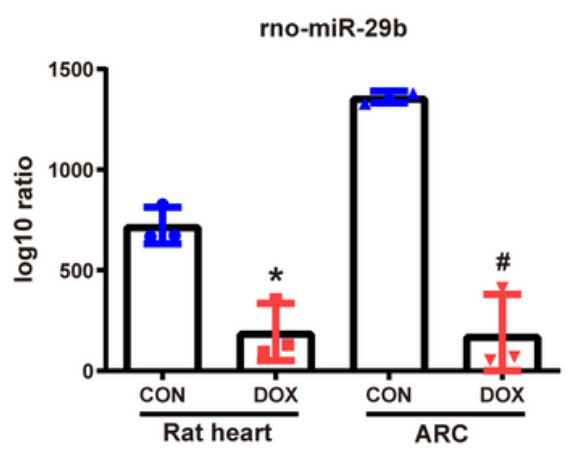

D

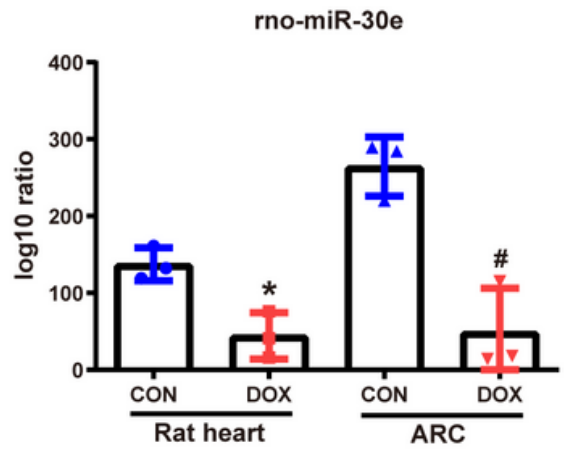

G

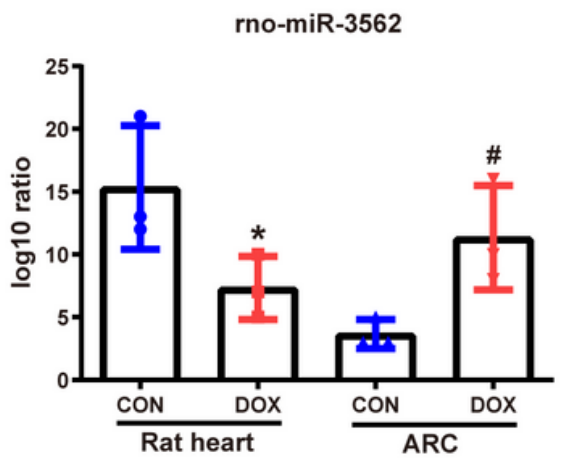

B

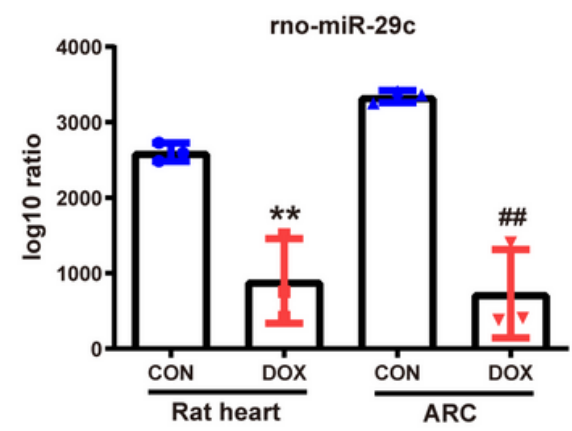

$\mathbf{E}$

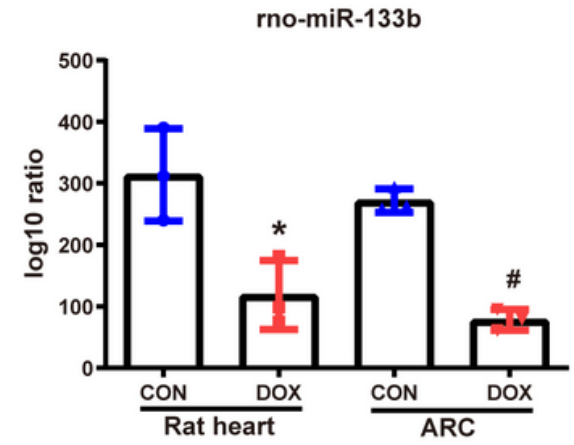

$\mathbf{H}$

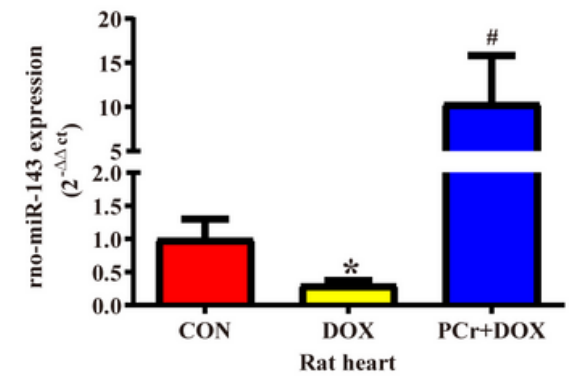

C

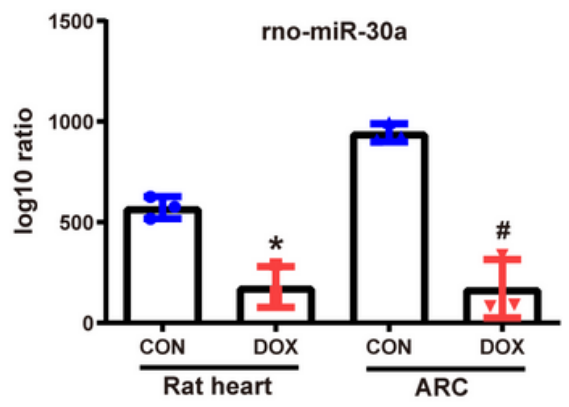

$\mathbf{F}$

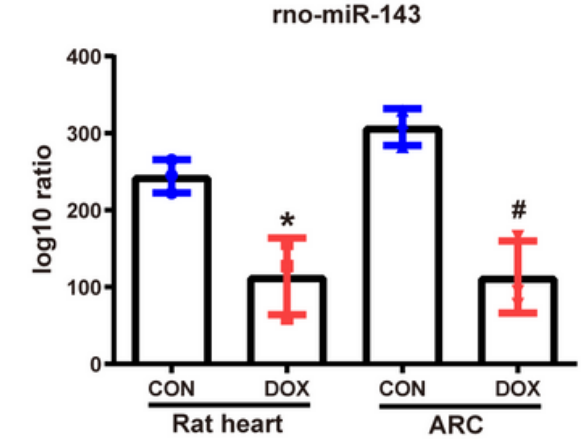

Figure 3

The expression analysis of DEMs and verification in DOX treatment rat. A-G The expression of DEMs in DOX-induced cardiotoxicity in vivo and in vitro from GSE36239 ( $n=3$ per group). $\mathbf{H}$ The expression of miR-143 in the heart tissue of DOX-induced cardiotoxicity with the intervention of $\mathrm{PCr}$ ( $n=6-8$ per group). All values were expressed as means \pm S.E.M. ${ }^{*} P<0.05$ vs control group, ${ }^{\sharp} P<0.05$ vs $D O X$ group. 


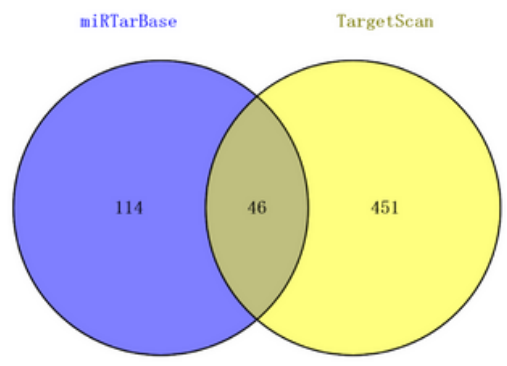

C

GO_CC

mitochondrial outer membrane |GO:0005741 dendrite cytoplasm [GO:0032839 intracellular membrane-bounded organelle [GO:0043231] cell-cell adherens junction |GO:0005913 receptor complex |GO:0043235
cytoplasm |GO:0005737| cytoplasm |GO:0005737|
intracellular |GO:0005622| Golgi apparatus |GO:0005794 cytosol [GO:0005829]

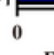

Enrichment Score (-log10(Pvalue))

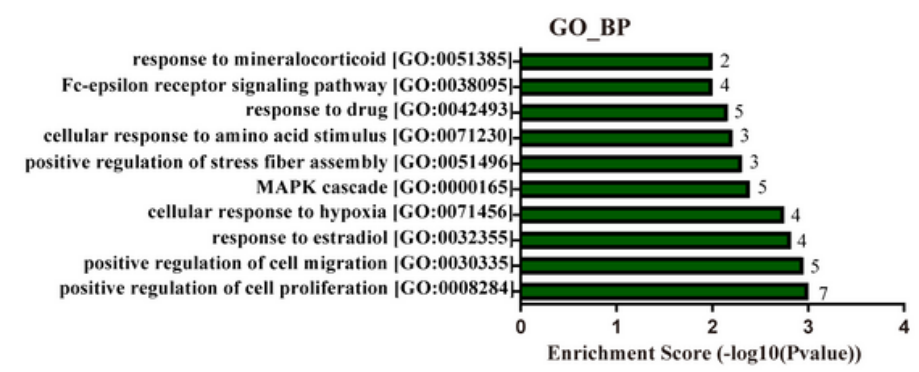

$\mathbf{E}$

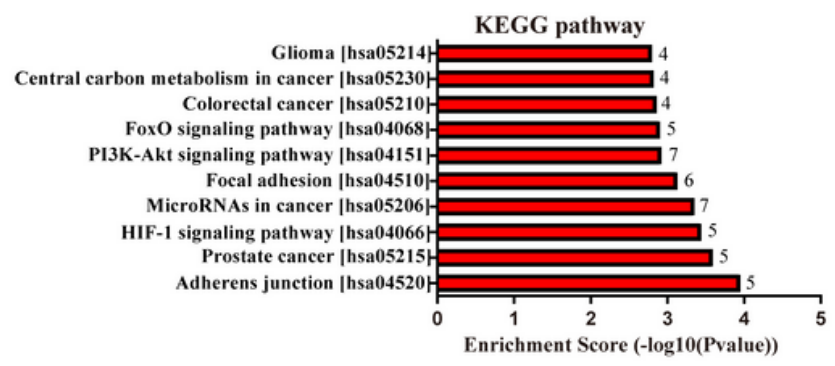

D

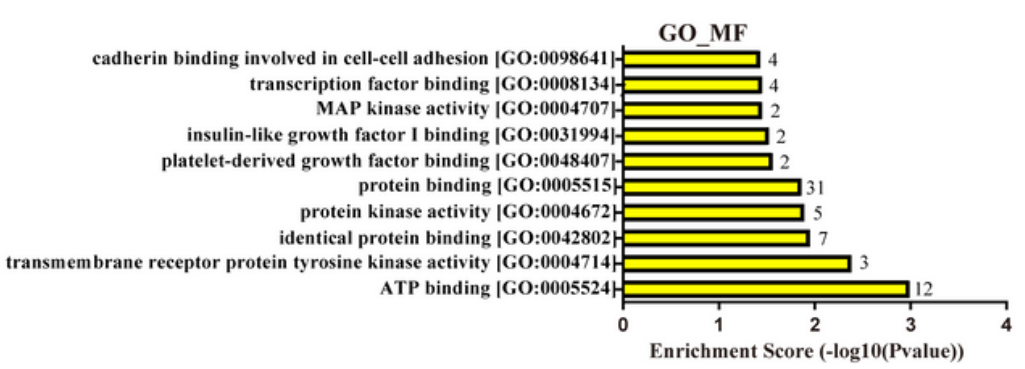

Figure 4

Prediction on target genes of miR-143, GO and KEGG pathway analysis. A Map the intersection of the target genes of miR-143 by Targetscan and miRBase in Species Homo sapiens by Venny 2.1. B The significantly enriched GO BP terms of DEMs. The $-\log _{10}(p$ value) indicate the Go score related to genes with biological process $\mathrm{P}$ value. C The significantly enriched GO CC terms of DEMs. The $-\log _{10}(p$ value) indicate the Go score related to genes with cellular component $P$ value. $D$ The significantly enriched GO MF terms of DEMs. The significantly enriched GO CC terms of DEMs. The - $\log _{10}(p$ value) indicate the Go score related to genes with molecular function $P$ value. $E$ The significantly enriched KEGG pathway of DEMs based on the KEGG database. The vertical axis represents the pathway category, the horizontal axis represents the $-\log _{10}(p$ value) of these significant pathways and the number on the right of the column represents the number of genes. 
A
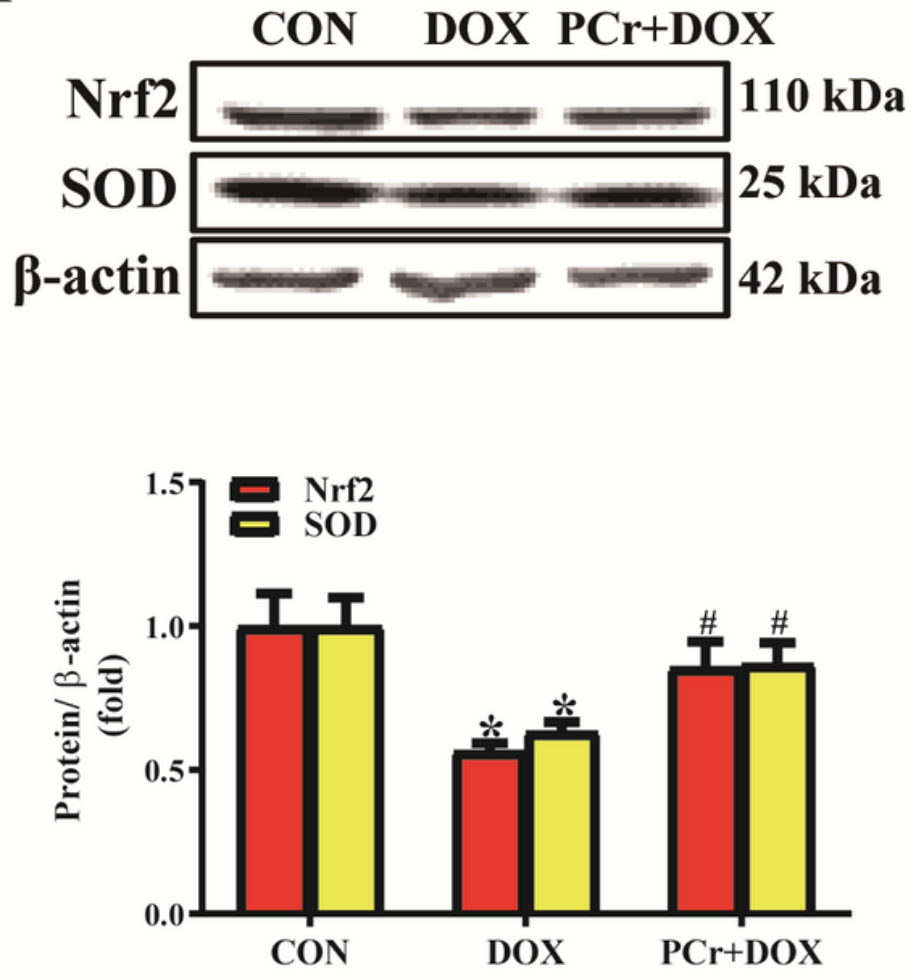

B
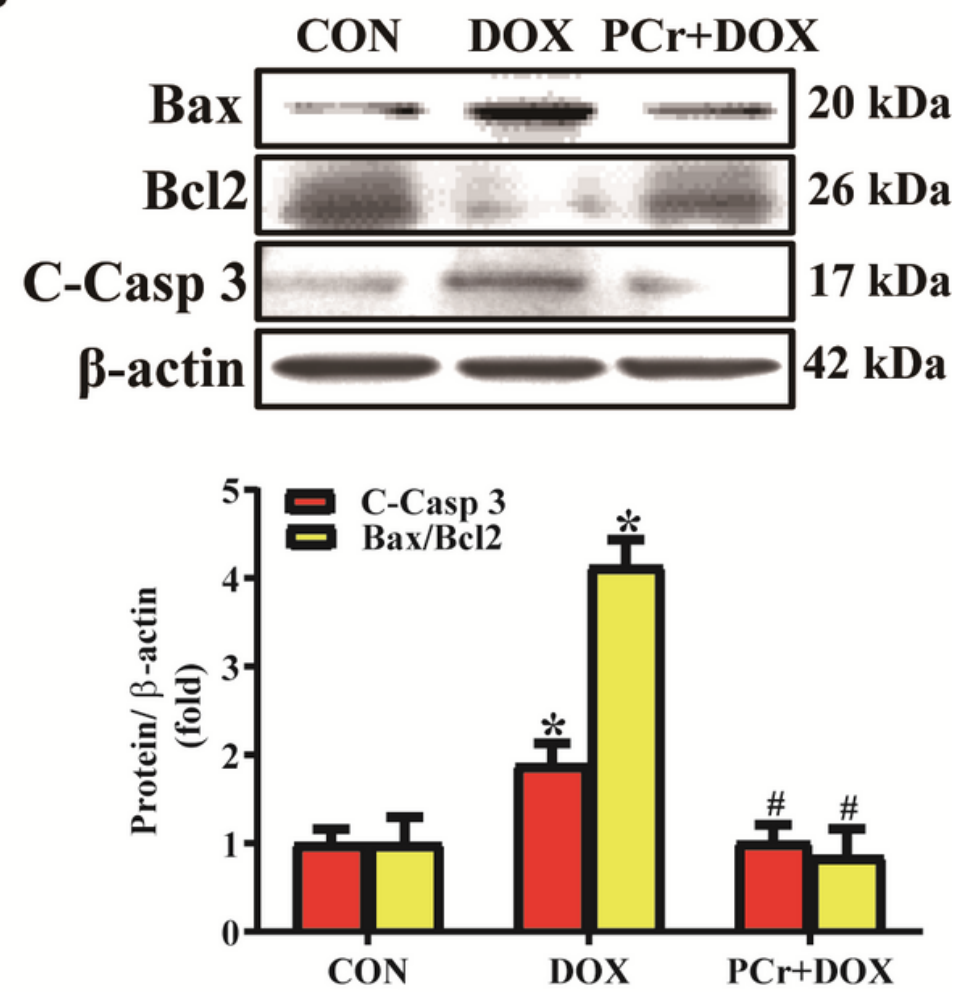

Figure 5

Effect of phosphocreatine ( $\mathrm{PCr}$ ) on the expression of miR-143 in rat heart tissues of DOX-induced cardiotoxicity. Western blot detected protein expression in DOX-treated $\mathrm{H} 9 \mathrm{c} 2$ cells with or without $\mathrm{PCr}$ pretreatment. Bottom: Semi-quantitative analysis of western blot. A Nrf2 and SOD, B Bax, Bcl2 and C-Casp 3. All values were expressed as means \pm S.E.M. $n=6-7$. ${ }^{*} P<0.05$ vs control group; ${ }^{\#} P<0.05$ vs DOX group.

A

B
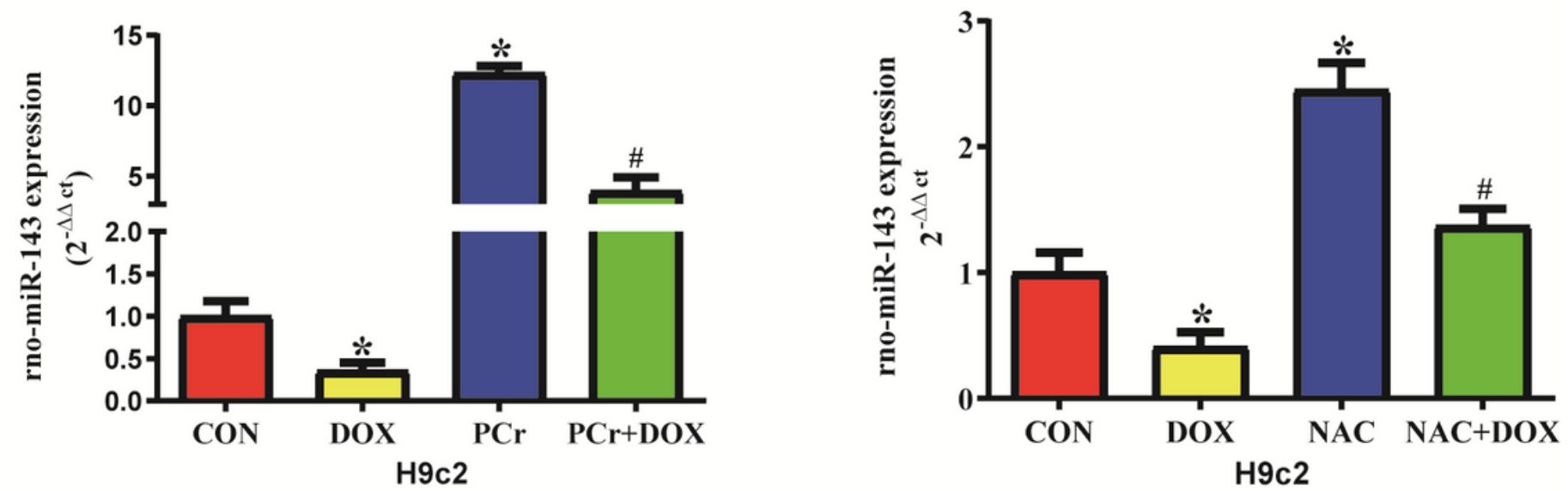

Figure 6

Effect of PCr or N-acetylcysteine (NAC) on the expression of miR-143 in H9c2 cells treated by DOX. A and B RT-PCR detected miR-143 expression. All values were expressed as means \pm S.E.M. $n=6-7$. ${ }^{*} P<0.05$ 
vs control group; ${ }^{\#} P<0.05$ vs DOX group.

\section{Supplementary Files}

This is a list of supplementary files associated with this preprint. Click to download.

- miR143targetpredictGOandKEGGanalysis.xIsx 